\title{
Fatores associados à adição de sal à refeição pronta
}

\author{
Factors associated with the addition of salt to prepared food
}

Raquel da Silva Assunção de Castro ${ }^{1}$

Luana Giatti ${ }^{2}$

Sandhi Maria Barreto ${ }^{3}$

${ }^{1}$ Programa de PósGraduação em Saúde Pública, Faculdade de Medicina, Universidade Federal de Minas Gerais (UFMG). Av. Prof. Alfredo Balena 190, Centro.

30.130-100 Belo Horizonte MG Brasil.

raquelac.ufmg@gmail.com ${ }^{2}$ Departamento de Nutrição Clínica e Social, Escola de Nutrição, Universidade Federal de Ouro Preto. ${ }^{3}$ Departamento de Medicina Preventiva e Social, Faculdade de Medicina, UFMG.
Abstract The scope of this research was to investigate the potential differences between men and women in the addition of salt to prepared food. The study included 47,557 individuals aged 18 to 64 participating in the Risk and Protection Factors for Chronic Disease Surveillance System by Telephone Interview carried out in 26 Brazilian state capitals and the Federal District in 2006. Differences between men and women were tested by the chi-square test and the association magnitudes between the dependent and independent variables were estimated by the Odds Ratio obtained by Multiple Logistic Regression analysis. The prevalence of the addition of salt to prepared food was $8.3 \%$, being higher among men (9,8\% vs $6,9 \%, p<0.01)$. After adjustment, the addition of salt to prepared food was higher in individuals with self-rated fair to poor health, reporting cardiovascular disease and living in the North of Brazil. Hypertensive individuals reported addition of less salt to prepared food. Educational level was not associated with salt usage. Men add more salt than women. Public health policies aimed at reducing salt intake by the population should take into account the gender differences in salt intake and the factors that contribute to such differences.

Key words Gender and health, Epidemiological surveys, Food consumption, Brazi, Sodium chloride in the diet, Telephone interview
Resumo O objetivo desta pesquisa foi investigar se existem diferenças na adição de sal à refeição pronta entre homens e mulheres. Foram incluidos 47.557 indivíduos de 18 a 64 anos, participantes do sistema de Vigilância de Fatores de Risco e Proteção para Doenças Crônicas por Inquérito Telefônico, das 26 capitais e Distrito Federal, em 2006. As diferenças entre homens e mulheres foram analisadas pelo teste do qui-quadrado e as magnitudes da associação estimadas pelo Odds Ratio obtido por meio da regressão logística múltipla. A prevalência da adição de sal à refeição pronta foi 8,3\%, sendo maior entre homens (9,8\% vs $6,9 \%, p<0,01)$. Após ajustes, a adição de sal à refeição pronta foi maior em indivíduos com a autoavaliação de saúde regular e ruim, relato de doença cardiovascular e que residiam na região Norte do Brasil. Indivíduos hipertensos relataram adicionar menos sal à refeição pronta. A escolaridade não foi associada à adição de sal. Homens adicionam mais sal à refeição pronta do que mulheres. Políticas públicas de saúde voltadas para a redução da ingestão de sal pela população devem levar em consideração a diferença de gênero no uso discricionário de sal e nos fatores que favorecem essas diferenças.

Palavras-chave Gênero e saúde, Inquéritos epidemiológicos, Consumo de alimentos, Brasil, Cloreto de sódio na dieta, Entrevista por telefone 


\section{Introdução}

Existem evidências de que a dieta rica em sal está associada à elevação da pressão arterial, à incidência de acidente vascular cerebral e de doença cardiovascular ${ }^{1,2}$. A redução moderada da ingestão de sal diminuiu a pressão sistólica e diastólica, ao longo de várias semanas até alguns anos de seguimento em estudos de intervenção, tanto em hipertensos como em normotensos. De acordo com uma metanálise com 32 ensaios clínicos, as reduções médias da pressão sistólica induzida pelo menor consumo de sódio em hipertensos e normotensos, foram, respectivamente, 4,8 e 1,9 mmHg e da pressão diastólica 2,5 e 1,1 mmHg ${ }^{3}$. Um ensaio clínico randomizado no nordeste do Japão mostrou que, em indivíduos saudáveis, o aconselhamento nutricional, juntamente com a redução da ingestão de sódio resultou em uma queda de $2,7 \mathrm{mmHg}$ na pressão sistólica no grupo de intervenção, comparado a um aumento de $0,5 \mathrm{mmHg}$ no grupo controle. A mesma intervenção em indivíduos hipertensos levou a uma diminuição ainda maior na pressão sistólica (5,6 $\mathrm{mmHg}$ ), comparado a um aumento de 1,4 $\mathrm{mmHg}$ no grupo controle $\mathrm{e}^{4} \mathrm{O}$ sal também pode ter efeito, independente da hipertensão, na ocorrência do acidente vascular cerebral, doença cardiovascular e progressão da doença renal crôni$\mathrm{ca}^{1}$. Além disso, evidências apontam que o consumo de sal pode estar indiretamente relacionado à obesidade, bem como ao aumento do risco de diabetes tipo 2 , independente de potenciais fatores de confusão, como inatividade física, obesidade e hipertensão $0^{1,5}$.

A hipertensão arterial é a principal causa de doença cardiovascular em países desenvolvidos, respondendo por $62 \%$ dos acidentes vasculares encefálicos e $49 \%$ das doenças coronarianas ${ }^{6}$. Segundo a Organização Mundial de Saúde (OMS) ${ }^{7}$, as doenças cardiovasculares são a principal causa de morte em pessoas com mais de 60 anos e a segunda causa de morte em pessoas de 15-49 anos.

Entre as estratégias apontadas para redução da doença cardiovascular em todo o mundo, a redução do sal é a mais custo-efetiva ${ }^{7}$. A OMS recomenda o consumo diário de menos de $5 \mathrm{~g}$ de sal (equivalente a $2 \mathrm{~g}$ de sódio), incluindo a ingestão de sódio de todas as fontes alimentares ${ }^{7,8}$. Pesquisa em 23 países estimou que a redução da ingestão de sal para o limite recomendado pela OMS poderá, em 10 anos, prevenir 8,5 milhões de mortes no mundo?.

Entretanto, apesar dessas evidências, a ingestão diária de sal é elevada e acima da recomenda- da na grande maioria das populações ${ }^{10}$. No Brasil, a quantidade de sódio disponível para consumo nos domicílios foi de $4,5 \mathrm{~g}$ por pessoa, excedendo, assim, em mais de duas vezes o limite recomendado de sódio ${ }^{11}$. Estudo publicado recentemente pela Organização Pan Americana de Saúde sobre o consumo de sal nas Américas estimou o mesmo em 11 gramas/dia no Brasil, 12 gramas/dia na Argentina, 9 gramas/dia no Chile, 8,7 gramas/dia nos Estados Unidos. De acordo com este mesmo estudo, em partes do Brasil, $70 \%$ do sal ingerido na dieta advém da adição discricionária de sal durante a preparação dos alimentos ou à mesa ${ }^{12}$.

Em geral, a adição de sal à refeição pronta é maior em homens que em mulheres ${ }^{13,14}$. Em estudo realizado nos Estados Unidos, a adição de sal aos alimentos prontos esteve associada ao padrão de baixa ingestão de frutas e verduras e ao padrão de elevada ingestão de carnes vermelhas ${ }^{15}$. Estes achados podem refletir a tendência à aglomeração dos comportamentos de risco para a saúde. Neumann et al. ${ }^{16}$, identificaram quatro padrões alimentares na população brasileira pela análise fatorial, um deles foi o padrão aterogênico composto por gorduras saturadas, adição de sal à refeição pronta e bebidas alcoólicas. Esse padrão predominou entre homens, esteve associado à menor escolaridade e ao tabagismo, entre outros fatores. Outro estudo apontou que o consumo diário de sal, estimado pela quantidade consumida por pessoa mensalmente no domicílio, foi maior em classes socioeconômicas mais baixas ${ }^{17}$.

O presente estudo considera que a adição de sal à refeição pronta é um indicador de maior consumo de sal e tem como hipótese que homens apresentam maior frequência de adição de sal do que mulheres. Tal hipótese foi investigada a partir do marcador de padrão alimentar "adição de sal à refeição pronta" presente no questionário do Vigitel em $2006^{18}$.

\section{Métodos}

Trata-se de um estudo transversal realizado com dados do sistema de Vigilância e Fatores de Risco e Proteção para Doenças Crônicas por Inquérito Telefônico (VIGITEL-2006) ${ }^{18}$. O VIGITEL é um sistema de monitoramento, implantado pelo Ministério da Saúde em 2006. Seu objetivo é monitorar a frequência e a distribuição de fatores de risco e proteção para doenças crônicas não transmissíveis nas 26 capitais brasileiras e no Dis- 
trito Federal. Utiliza para isso amostras probabilísticas da população de adultos, servidos por, pelo menos, uma linha de telefone fixa no ano.

O tamanho amostral mínimo estabelecido foi de 2000 indivíduos por cidade com 18 ou mais anos de idade para estimar a frequência de qualquer fator de risco na população adulta, com o nível de confiança de 95\% e erro máximo de dois pontos percentuais ${ }^{19}$.

A primeira etapa do processo consistiu do sorteio sistemático de 5000 linhas telefônicas por cidade, estratificado por região ou prefixo, realizado a partir do cadastro eletrônico das linhas residenciais fixas. A seguir, as linhas foram ressorteadas e divididas em 25 réplicas de 200 linhas ${ }^{19}$.

A segunda etapa do processo envolveu a identificação das linhas residenciais ativas e o sorteio de um indivíduo por domicílio para participar da entrevista. O número total de adultos entrevistados pelo VIGITEL em 2006 foi de 54.369, sendo 21.294 homens e 33.075 mulheres. A realização do VIGITEL foi aprovada pelo Comitê Nacional de Ética em Pesquisa para Seres Humanos do Ministério da Saúde ${ }^{18}$. Maiores detalhes sobre os procedimentos estão descritos por Moura et al. ${ }^{19}$.

A população do presente estudo foi de 47.557 indivíduos de 18 a 64 anos de idade participantes do VIGITEL no ano de 2006. Foram excluídos os indivíduos com idade igual ou superior a 65 anos porque a maior frequência de problemas de saúde nessa faixa etária pode modificar a adição de sal à refeição pronta. Também foram excluídas as mulheres que relataram gravidez atual, devido às mudanças dos hábitos alimentares e do Índice de Massa Corporal (IMC) nesse período.

A variável dependente pesquisada foi: adição de sal à refeição pronta. A variável foi criada a partir da resposta à pergunta: “O $\operatorname{sr}(\mathrm{a})$ costuma adicionar sal na comida pronta, no seu prato, sem contar a salada?". As alternativas de respostas eram: não; sim, de vez em quando e sim. A variável foi categorizada em sim e não. Considerou-se como sim as respostas "sim" e "sim, de vez em quando".

A variável explicativa de interesse neste estudo foi sexo, as demais foram consideradas potenciais fatores de confusão na associação entre essas e a variável resposta.

As variáveis independentes foram agrupadas em sociodemográficas, comportamentais e de saúde. As sociodemográficas foram: sexo (masculino; feminino), estado civil (casado/juntado; solteiro; separado/divorciado/viúvo) faixa etária em anos $(18-24 ; 25-34 ; 35-44 ; 55-64)$, escola- ridade em anos de estudo (0-8; 9-11; 12 e mais), região de moradia, obtida pelo agrupamento das capitais brasileiras em regiões (sudeste; nordeste; sul; norte; centro-oeste). As variáveis comportamentais e de saúde foram: índice de massa corporal (IMC) referido, criado a partir do peso e estatura (normal " $<25 \mathrm{~kg} / \mathrm{m}^{2 \text { "; }}$ sobrepeso " 25 a $29,9 \mathrm{~kg} / \mathrm{m}^{2 \text { " }}$ e obesidade " $\geq 30 \mathrm{~kg} / \mathrm{m}^{2 "}$ ), relato de fazer dieta atualmente (sim; não), autoavaliação de saúde (excelente/bom; regular; ruim), atividade física no lazer ( sim; não), relato de diabetes ( sim; não), relato de hipertensão ( sim; não), relato de dislipidemias ( $\operatorname{sim}$; não) e relato de doença cardiovascular (sim; não).

Considerou-se atividade física no lazer a prática de pelo menos 30 minutos diários de atividade física de intensidade leve ou moderada (caminhada, caminhada em esteira, musculação, hidroginástica, ginástica em geral, natação, artes marciais, ciclismo e voleibol) em cinco ou mais dias da semana ou a prática de pelo menos 20 minutos diários de atividade física de intensidade vigorosa (corrida, corrida em esteira, ginástica aeróbica, futebol, basquetebol e tênis) em três ou mais dias da semana.

As variáveis hipertensão, diabetes, doença cardiovascular (infarto, derrame ou acidente vascular cerebral) e dislipidemias (colesterol ou triglicérides elevados) foram definidas com base no relato do diagnóstico médico destas doenças pela seguinte pergunta: "Algum médico já lhe disse que o(a) $\operatorname{sr}(\mathrm{a})$ tem...?”

Realizou-se a análise descritiva da população de estudo. As diferenças entre homens e mulheres foram verificadas pelo teste do qui-quadrado, com nível de significância de 0,05. A magnitude da associação entre a variável dependente e cada variável independente foi estimada pelo odds ratio por meio de regressão logística com intervalos de confiança (IC) de 95\%. A análise multivariada foi realizada em duas etapas. Inicialmente foram incluídas as variáveis que apresentaram $\mathrm{p}<0,20$ na análise univariada. $\mathrm{O}$ modelo final foi estimado com as variáveis que apresentaram $\mathrm{p}<0,05$ nas análises intermediárias.

Utilizou-se o Software Stata (versão 10.0) para realização das análises. Todas as estimativas levaram em conta três fatores de ponderação para correção do processo amostral. Esses fatores visavam: 1) corrigir a maior chance dos indivíduos de domicílios com mais de uma linha telefônica serem selecionados; 2) a menor chance dos indivíduos de domicílios habitados por mais pessoas serem selecionados; 3) igualar a composição sociodemográfica da amostra de adultos em 
cada cidade à composição sociodemográfica da população adulta total da cidade ${ }^{19}$.

\section{Resultados}

Dos 47.557 participantes, 52,6\% eram mulheres e $47,4 \%$ eram homens. A idade média foi de 38,2 $( \pm 12,6)$ anos, sendo que $27,4 \%$ tinham entre 25 a 34 anos e $23,6 \%$ tinham entre 35 a 44 anos. A maioria era casado ou vivia junto. Cerca de 50,7\% apresentavam escolaridade abaixo de oito anos de estudo. Entre as regiões de moradia pesquisadas, a região Sudeste foi a que apresentou maior proporção de indivíduos (47,6\%).

$\mathrm{A}$ adição de sal à refeição pronta foi relatada por $8,3 \%$ dos indivíduos. A maioria relatou não fazer dieta para restrição de peso $(86 \%)$ e não praticar atividade física no lazer $(84,8 \%)$. Quase metade da população apresentou obesidade e sobrepeso, sendo estes percentuais iguais a $18,0 \%$ e 28,7\%, respectivamente. A autoavaliação de saúde foi considerada boa ou excelente por $66 \%$ dos indivíduos. Cerca de $4 \%$ relatou diabetes, 18,2\%, hipertensão, 14,8\%, dislipidemias e $2 \%$, doença cardiovascular.

Houve maior adição de sal à refeição pronta pelos homens $(9,8 \%$ vs $6,9 \% ; \mathrm{p}<0,01)$. As mulheres relataram maior prevalência de obesidade, fazer mais dieta atualmente e pior autoavaliação de saúde que os homens. Enquanto eles apresentaram mais sobrepeso, maior prática de atividade física no lazer e menores prevalências de diabetes, hipertensão e dislipidemias (Tabela 1).

$\mathrm{Na}$ análise univariada, homens adicionavam sal à refeição pronta com maior frequência do que as mulheres, solteiros, assim como as pessoas que autoavaliaram a saúde como regular e que residiam na região Norte. A adição de sal diminuiu com o aumento da faixa etária e com o relato de hipertensão (Tabela 2).

$\mathrm{Na}$ análise multivariada, a frequência da adição de sal à refeição pronta foi maior nos homens, nos solteiros, nas pessoas que autoavaliaram a saúde como regular e ruim, relataram doença cardiovascular e residiam na região Norte. Essa proporção reduziu com o relato de hipertensão arterial. (Tabela 3).

\section{Discussão}

Os resultados mostram que os homens adicionam sal à refeição pronta mais frequentemente do que as mulheres. Essa diferença de compor- tamento entre homens e mulheres permanece após ajustes por potenciais fatores de confusão.

No presente estudo, assumimos que o indicador de adição de sal à refeição pronta está associado ao maior consumo desse produto. Diversos estudos publicados recentemente também utilizaram este indicador para estudar a associação entre a ingestão de sódio e a ocorrência de problemas de saúde ${ }^{20-23}$. Entretanto, é importante ressaltar que existem várias fontes de sal, sendo à adição de sal uma fonte menor para a ingestão total de sódio, sobretudo nos países desenvolvidos da Europa e América do Norte ${ }^{10}$. Na Inglaterra, uma pesquisa na década de 80 mostrou que a fonte de sal discricionária, no preparo dos alimentos ou à mesa, contribuía com apenas 15\% do total de sal ingerido pelos britânicos. Ou seja, o sal proveniente de alimentos manufaturados ou preparados para venda respondia por $85 \%$ do consumo total de sódio ${ }^{24}$. Mas estudo recente estima que, no Japão e na China, o sal adicionado à mesa e na cozinha, bem como os molhos de soja, representam as maiores fontes de ingestão de sal ${ }^{10}$. No Brasil, a maior parte do sódio disponível para consumo nos domicílios provém do sal de cozinha e de condimentos à base de sal (76,2\%), embora a fração proveniente de alimentos processados com adição de sal aumente linear e intensamente com o poder aquisitivo domiciliar $^{11}$. Com o crescimento do consumo de alimentos processados e da refeição fora de casa nos grupos socioeconômicos mais pobres ${ }^{25}$, é possível que a contribuição do sal usado no cozimento ou adicionado à mesa para a ingestão total de sódio tenha decaído. Os gastos com alimentação fora do domicílio, que correspondiam a $24,1 \%$ em $2002-2003$, elevaram-se para $31,1 \%$ em 2008-2009. O consumo de alimentos prontos e misturas industrializadas tiveram um aumento relativo de $40 \%$ nesse período ${ }^{26}$. Apesar disso, e devido a enorme dificuldade de mensurar o sal na dieta em grandes inquéritos de saúde, acreditamos que o indicador de adição de sal à comida pronta tem importante correlação positiva com o maior consumo geral de sódio e é corroborada por estudo anterior ${ }^{27}$.

Estudos em outros países também mostraram maior adição de sal à refeição pronta por parte dos homens ${ }^{13,14}$. Análise realizada na Inglaterra mostrou que os homens ingeriam mais sal adicionado à refeição do que as mulheres $(0,77$ $\mathrm{g} /$ dia vs $0,46 \mathrm{~g} / \mathrm{dia}$, respectivamente $)^{24}$. Esses achados são importantes, pois homens têm maior risco de doença cardiovascular e renal que mulheres na pré-menopausa. Estudos apontam que 
Tabela 1. Qui-quadrado segundo aspectos de consumo alimentar, sociodemográficos, comportamentais e de saúde da população de estudo por sexo. Brasil, Vigitel, 2006. $(\mathrm{n}=47.557)$

\begin{tabular}{|c|c|c|c|}
\hline Variável & $\begin{array}{c}\text { Sexo Masculino } \\
(\%)\end{array}$ & $\begin{array}{c}\text { Sexo Feminino } \\
(\%)\end{array}$ & Valor de $p$ \\
\hline \multicolumn{4}{|l|}{ Adição de sal à refeicão pronta } \\
\hline Sim & 9,8 & 6,9 & $<0,01$ \\
\hline Não & 90,2 & 93,1 & \\
\hline \multicolumn{4}{|l|}{ Faixa etária } \\
\hline 18-24 anos & 24,0 & 22,1 & \\
\hline 25-34 anos & 28,0 & 26,9 & 0,05 \\
\hline $35-44$ anos & 23,2 & 23,9 & \\
\hline 45-54 anos & 15,7 & 16,8 & \\
\hline $55-64$ anos & 9,0 & 10,3 & \\
\hline \multicolumn{4}{|l|}{ Escolaridade em anos ${ }^{*}$} \\
\hline$<5$ anos & 16,3 & 18,0 & \\
\hline$\geq 5$ e $<9$ anos & 36,5 & 32,5 & $<0,01$ \\
\hline$\geq 9 \mathrm{e}<12$ anos & 29,4 & 31,8 & \\
\hline$\geq 12$ anos & 17,8 & 17,8 & \\
\hline \multicolumn{4}{|l|}{ Estado civil } \\
\hline Solteiro & 40,9 & 37,6 & \\
\hline Casado/Juntado & 53,9 & 51,2 & $<0,01$ \\
\hline Separado/Divorciado/Viúvo & 5,1 & 11,2 & \\
\hline \multicolumn{4}{|l|}{ IMC referido } \\
\hline$<25 \mathrm{~kg} / \mathrm{m}^{2}$ & 51,1 & 55,4 & $<0,01$ \\
\hline$\geq 25 \mathrm{e}<30 \mathrm{~kg} / \mathrm{m}^{2}$ & 34,6 & 23,4 & \\
\hline$\geq 30 \mathrm{~kg} / \mathrm{m}^{2}$ & 14,4 & 21,3 & \\
\hline \multicolumn{4}{|l|}{ Relato de dieta atual } \\
\hline Sim & 8,7 & 18,8 & $<0,01$ \\
\hline Não & 91,3 & 81,2 & \\
\hline \multicolumn{4}{|l|}{ Autoavaliação de saúde $e^{* *}$} \\
\hline Excelente/Bom & 71,2 & 61,5 & \\
\hline Regular & 24,8 & 32,7 & $<0,01$ \\
\hline Ruim & 4,1 & 5,8 & \\
\hline \multicolumn{4}{|l|}{ Atividade física no lazer } \\
\hline Sim & 18,4 & 12,3 & $<0,01$ \\
\hline Não & 81,6 & 87,7 & \\
\hline \multicolumn{4}{|l|}{ Diabetes } \\
\hline Sim & 3,5 & 4,5 & 0,01 \\
\hline Não & 96,6 & 95,5 & \\
\hline \multicolumn{4}{|l|}{ Hipertensão } \\
\hline Sim & 15,7 & 20,5 & $<0,01$ \\
\hline Não & 84,3 & 79,6 & \\
\hline \multicolumn{4}{|l|}{ Doença Cardiovascular ${ }^{* * *}$} \\
\hline $\operatorname{Sim}$ & 2,1 & 1,9 & 0,57 \\
\hline Não & 97,9 & 98,1 & \\
\hline \multicolumn{4}{|l|}{ Dislipidemias $^{* * * *}$} \\
\hline Sim & 13,7 & 15,8 & $<0,01$ \\
\hline Não & 86,3 & 84,2 & \\
\hline \multicolumn{4}{|l|}{ Região de residência } \\
\hline Sudeste & 47,8 & 47,4 & 0,56 \\
\hline Nordeste & 23,9 & 25,0 & \\
\hline Sul & 8,4 & 8,4 & \\
\hline Norte & 9,1 & 8,6 & \\
\hline Centro-oeste & 10,7 & 10,6 & \\
\hline
\end{tabular}

Não informados: ${ }^{*}=599 ;{ }^{* *}=120 ;{ }^{* * *}=9 ;{ }^{* * * *}=284$. 
Tabela 2. Análise univariada de características associadas à adição de sal à refeição pronta da população de estudo, Brasil, Vigitel, 2006. ( $\mathrm{n}=47.557)$

\begin{tabular}{|c|c|c|}
\hline Variável & $\begin{array}{l}\text { Odds } \\
\text { ratio }\end{array}$ & $\begin{array}{c}\text { Intervalo de } \\
\text { Confiança }\end{array}$ \\
\hline \multicolumn{3}{|l|}{ Sexo } \\
\hline Feminino & 1 & - \\
\hline Masculino & 1,47 & $1,26-1,71$ \\
\hline \multicolumn{3}{|l|}{ Faixa etária } \\
\hline 18-24 anos & 1 & - \\
\hline 25-34 anos & 0,82 & $0,66-1,03$ \\
\hline $35-44$ anos & 0,65 & $0,52-0,80$ \\
\hline $45-54$ anos & 0,62 & $0,49-0,80$ \\
\hline 55-64 anos & 0,57 & $0,42-0,78$ \\
\hline \multicolumn{3}{|l|}{ Estado civil } \\
\hline Casado/Juntado & 1 & - \\
\hline Solteiro & 1,52 & $1,30-1,79$ \\
\hline Separado/Divorciado/Viúvo & 0,84 & $0,63-1,10$ \\
\hline \multicolumn{3}{|l|}{ Região de residência } \\
\hline Sudeste & 1 & - \\
\hline Nordeste & 1,67 & $0,98-1,40$ \\
\hline Sul & 0,86 & $0,68-1,09$ \\
\hline Norte & 1,50 & $1,24-1,82$ \\
\hline Centro-oeste & 0,98 & $0,75-1,27$ \\
\hline \multicolumn{3}{|l|}{ IMC referido } \\
\hline$<25 \mathrm{~kg} / \mathrm{m}^{2}$ & 1 & - \\
\hline$\geq 25$ e $<30 \mathrm{~kg} / \mathrm{m}^{2}$ & 0,95 & $0,79-1,14$ \\
\hline$\geq 30 \mathrm{~kg} / \mathrm{m}^{2}$ & 0,85 & $0,67-1,07$ \\
\hline \multicolumn{3}{|l|}{ Relato de dieta atual } \\
\hline Não & 1 & - \\
\hline Sim & 0,86 & $0,68-1,08$ \\
\hline \multicolumn{3}{|l|}{ Autoavaliação de saúde ${ }^{*}$} \\
\hline Excelente/Bom & 1 & - \\
\hline Regular & 1,19 & $1,00-1,41$ \\
\hline Ruim & 1,35 & $0,91-2,02$ \\
\hline \multicolumn{3}{|l|}{ Atividade física no lazer } \\
\hline Não & 1 & - \\
\hline Sim & 1,05 & $0,83-1,33$ \\
\hline \multicolumn{3}{|l|}{ Diabetes } \\
\hline Não & 1 & - \\
\hline $\operatorname{Sim}$ & 0,90 & $0,49-1,66$ \\
\hline \multicolumn{3}{|l|}{ Hipertensão } \\
\hline Não & 1 & \\
\hline Sim & 0,58 & $0,45-0,75$ \\
\hline \multicolumn{3}{|l|}{ Doença Cardiovascular ${ }^{* *}$} \\
\hline Não & 1 & - \\
\hline Sim & 1,61 & $0,94-2,73$ \\
\hline \multicolumn{3}{|l|}{ Dislipidemias $^{* \star *}$} \\
\hline Não & 1 & - \\
\hline $\operatorname{Sim}$ & 0,96 & $0,76-1,22$ \\
\hline
\end{tabular}

Não informados: ${ }^{*}=120 ;{ }^{* *}=9 ;{ }^{* * *}=284$.

a pressão arterial é maior em homens que em mulheres em idades similares, entretanto após a menopausa, eleva-se mais em mulheres do que
Tabela 3. Análise multivariada de características associadas à adição de sal à refeição pronta da população de estudo, Brasil, Vigitel, 2006. ( $\mathrm{N}=$ 47.429)

\begin{tabular}{lcc}
\hline \multicolumn{1}{c}{ Variável } & $\begin{array}{c}\text { Odds } \\
\text { ratio }\end{array}$ & $\begin{array}{c}\text { Intervalo de } \\
\text { Confiança }\end{array}$ \\
\hline $\begin{array}{l}\text { Sexo } \\
\text { Feminino } \\
\text { Masculino }\end{array}$ & 1 & - \\
Estado civil & 1,47 & $1,26-1,72$ \\
$\quad$ Casado/Juntado & 1 & - \\
$\quad$ Solteiro & 1,47 & $1,24-1,74$ \\
$\quad$ Separado/Divorciado/Viúvo & 0,90 & $0,67-1,20$ \\
Região de residência & & \\
$\quad$ Sudeste & 1 & - \\
$\quad$ Nordeste & 1,11 & $0,93-1,33$ \\
Sul & 0,87 & $0,69-1,11$ \\
$\quad$ Norte & 1,42 & $1,17-1,73$ \\
Centro-oeste & 0,97 & $0,74-1,26$ \\
Autoavaliação de saúde & & \\
$\quad$ Excelente/Bom & 1 & - \\
Regular & 1,34 & $1,12-1,60$ \\
Ruim & 1,62 & $1,08-2,41$ \\
Hipertensão & & \\
Não & 1 & - \\
Sim & 0,57 & $0,43-0,74$ \\
Doença Cardiovascular & & \\
Não & 1 & - \\
Sim & 2,08 & $1,20-3,59$ \\
\hline
\end{tabular}

em homens ${ }^{28}$. Além disso, homens têm menor controle da hipertensão do que mulheres ${ }^{29}$.

O maior consumo de sal entre homens parece relacionado à maior ingestão energética e proteica masculina ${ }^{2,10}$. As carnes processadas constituem uma das maiores fontes de sódio alimentar sob a forma de cloreto de sódio $(\mathrm{sal})^{30}$. Homens consomem carne vermelha com mais frequência, principalmente salsicha e carne de porco, e alimentos ricos em amido, como batatas e pães $^{31}$. Além disso, apresentam outros comportamentos não saudáveis, como tabagismo, maior consumo de refrigerantes e bebidas alcoóli$\mathrm{cas}^{32}$ e provavelmente frequentam mais bares e restaurantes, o que levaria ao maior consumo de aperitivos, desenvolvendo maior preferência por alimentos salgados que as mulheres. As preferências masculinas podem favorecer a maior adição de sal à refeição pronta, já que aperitivos são frequentemente salgados à mesa, como a batata frita. A maioria deles tem pouco conhecimento sobre a importância da alimentação na prevenção de doenças, que é essencial para melhoria 
das práticas alimentares e redução dos riscos à saúde. Por outro lado, cozinhar e nutrição são construções sociais do gênero feminino ${ }^{33}$. As mulheres tendem a valorizar mais a importância da escolha dos alimentos para a saúde, o que poderia explicar um menor consumo de sal por parte das mesmas. Em estudo com jovens adultos em 23 países, as diferenças de gênero em relação ao sal foram explicadas principalmente pela maior aderência das mulheres à alimentação saudável ${ }^{14}$. As mulheres também são mais interessadas em questões nutricionais que os homens e tendem a apresentar maior conhecimento nutricional que estes, provavelmente pelo seu maior envolvimento com a alimentação ao longo da vida, devido ao seu papel social de cuidadora dentro e fora do $\operatorname{lar}^{31,33}$.

Em nosso estudo não encontramos associação entre escolaridade e adição de sal à refeição pronta. Molina et al. ${ }^{17}$ encontraram que a excreção urinária de sódio, bem como a quantidade de sal consumida no domicílio era mais alta em indivíduos de piores condições socioeconômicas, geralmente de menor escolaridade. Purdy et al. ${ }^{34}$ pesquisaram a influência socioeconômica sobre o consumo de sal e também encontraram maior uso discricionário de sal e frequente consumo de alimentos processados entre consumidores de baixo nível socioeconômico. É possível que a diferença socioeconômica encontrada por estes autores seja explicada por outras fontes de consumo de sal, discricionárias ou não, como a quantidade utilizada no preparo dos alimentos e o consumo de produtos industrializados.

A associação da adição de sal à refeição pronta com o estado civil solteiro é esperada, já que o casamento tem sido associado positivamente com o comportamento alimentar em homens e mulheres. Homens teriam maior vantagem sobre sua saúde com o casamento, provavelmente porque se beneficiam do cuidado em saúde prestado pelas mulheres aos outros membros da família ${ }^{35}$.

As diferenças regionais observadas na adição de sal à refeição pronta parecem ser influenciadas pela disponibilidade do sal nas regiões do Brasil, além das diferenças culturais. Dados da POF de 2002 e 2003 mostraram maior aquisição domiciliar per capita anual de sal nas regiões Norte, Sul e Nordeste, e menor nas regiões Sudeste e Centro-Oeste ${ }^{36}$.

A associação entre a adição de sal à refeição e a autoavaliação de saúde regular e ruim pode ser explicada tanto pela influência direta dos comportamentos negativos sobre a avaliação de saúde como pela maior frequência de problemas de saúde entre os que apresentam pior dieta. Em estudo sobre comportamento saudável em adultos jovens no Brasil, utilizando dados do Vigitel, comportamentos menos saudáveis foram associados à autopercepção de saúde ruim ${ }^{37}$. Associação similar foi relatada em estudo com adultos na Irlanda ${ }^{38}$ e nos Estados Unidos ${ }^{39}$.

A associação entre adição de sal à refeição pronta e relato de doença cardiovascular pode explicitar o maior risco e o pior controle dessas doenças em indivíduos que apresentam uma dieta com alto teor de sódio, e também foi observada em outros estudos ${ }^{2,40}$. Por outro lado, esta associação é preocupante, pois sugere uma baixa aderência à recomendação de redução do sal entre indivíduos com doença cardiovascular. Entretanto, o mesmo não ocorre com relação ao relato de hipertensão, visto que o mesmo se associou negativamente à adição de sal à refeição, sugerindo aderência à recomendação de redução de sal na dieta. Ou seja, é bastante provável que esse achado reflita causalidade reversa, já que a restrição de sal é uma orientação dietética para pessoas com hipertensão. Em estudo com diabéticos e/ou hipertensos de um município brasileiro $97,2 \%$ dos entrevistados afirmaram não adicionar sal à comida preparada ${ }^{41}$. Barreto e Figueiredo $^{37}$ pesquisaram a associação entre doença crônica e comportamento de risco e verificaram que indivíduos que relataram doenças crônicas não transmissíveis tiveram menos comportamentos de risco, incluindo adicionar menos sal aos alimentos. É possível que a orientação de redução do sal na dieta seja menos enfatizada entre indivíduos que já apresentam doença cardiovascular. Tal achado merece investigação específica no país, já que sinaliza pior cuidado com a saúde entre aqueles que estão doentes. Nos Estados Unidos, entre 2005 e 2006, 1 em cada 10 adultos conhecia a recomendação da redução na ingestão de sódio. Adultos com recomendação expressa de ingestão de sódio $\leq 1500 \mathrm{mg} /$ dia consumiram mais que o dobro do limite recomendado ${ }^{42}$.

Estudos que utilizam entrevistas por telefone podem apresentar viés de aferição, já que indivíduos residentes em domicílios sem cobertura por telefonia fixa, geralmente pertencentes às classes sociais menos favorecidas, não são incluídos. Visando atenuar essa limitação foram adotados pesos pós-estratificação para corrigir ao menos parcialmente vieses determinados pelas diferenças nas taxas de cobertura por telefonia fixa ${ }^{19}$.

A preocupação em torno da elevada ingestão do sal tem se tornado um assunto discutido em nível mundial. Ainda mais quando se considera 
que a redução da ingestão do sal é a medida mais custo-efetiva para diminuir a mortalidade por doenças cardiovasculares. Muitos países, como, por exemplo, Finlândia e Reino Unido têm realizado programas de redução de sal com sucesso ${ }^{1}$. No Brasil, a disponibilidade de sódio para consumo ultrapassa em mais de duas vezes o recomendado, urgindo a implantação de políticas efetivas de redução da ingestão de sal no país.

As políticas públicas voltadas para reduzir a ingestão de sal por parte da população necessitam levar em consideração a diferença de gênero na adição de sal à refeição pronta mostradas no presente trabalho, entre outros fatores. Além de informar aos homens mais e melhor sobre os malefícios do sal, é fundamental abordar os aspectos culturais relacionados a este comportamento entre os mesmos. Entretanto, é funda- mental enfatizar que mudanças no comportamento alimentar são insuficientes para a redução do sal na alimentação no Brasil. Para se alcançar, de forma ampla e sustentável, a meta de redução do consumo de sal para menos de $5 \mathrm{~g}$ por pessoa por dia até 2020, é essencial que haja uma redução do sódio, presente nos alimentos industrializados ou já processados para a venda. Inúmeras iniciativas neste sentido já estão em discussão, como o imposto sobre o sódio, e algumas já foram implantadas no país, como o acordo com as indústrias de massas, panificadoras e confeitarias ${ }^{43}$. Espera-se que as intervenções já implantadas no Brasil sejam efetivas e que outras intervenções venham a se somar a estas, visando melhorar não só o padrão alimentar da população, mas também reduzir o risco das doenças crônicas não transmissíveis.

\section{Colaboradores}

RSA Castro participou no planejamento, análise e interpretação de dados, redação do artigo e elaboração e aprovação da versão final do manuscrito. SM Barreto participou no planejamento, análise e interpretação de dados, revisão crítica de seu conteúdo, elaboração e aprovação da versão final do manuscrito. L Giatti participou no planejamento, análise e interpretação de dados, revisão crítica de seu conteúdo, elaboração e aprovação da versão final do manuscrito. 


\section{Referências}

1. He FJ, MacGregor GA. Reducing population salt intake worldwide: from evidence to implementation. Prog Cardiovasc Dis 2010; 52(5):363-382.

2. Morrison AC, Ness RB. Sodium intake and cardiovascular disease. Annu Rev Public Health 2011; 32:7190.

3. Cuttler JA, Follmann D, Allender DS. Randomized trials of sodium reduction: an overview. Am J Clin Nutr 1997: 65(Supl. 2):643S-651S.

4. Takahashi Y, Sasaki S, Okubo S, Hayashi M, Tsugane S. Blood pressure change in a free-living population-based dietary modification study in Japan. $J$ Hypertens 2006; 24(3):451-458.

5. Hu G, Jousilahti P, Peltonen M, Lindström J. Urinary sodium and potassium excretion and the risk of type 2 diabetes: a prospective study in Finland. Diabetologia 2005; 48(8):1477-1483.

6. He FJ, McGregor GA. Salt, blood pressure and cardiovascular disease. Curr Opin Cardiol 2007; 22(4): 298-305.

7. World Health Organization (WHO). Reducing salt intake in populations: Report of a WHO Forum and Technical Meeting 5-7 October 2006, Paris. Geneva: WHO; 2007.

8. World Health Organization (WHO). Diet, nutrition and prevention of chronic diseases: Report of a Joint WHO/FAO Expert Consultation. Geneva: WHO; 2003.

9. Asaria P, Chisholm D, Mathers C, Ezzati M, Beaglehole R. Chronic disease prevention: health effects and financial costs of strategies to reduce salt intake and control tabacco use. Lancet 2007; 370(9604): 2044-2053.

10. Brown IJ, Tzoulaki I, Candeias V, Elliott P. Salt intake around the world: implications for public health. Int J Epidemiol 2009; 38(3):791-813.

11. Sarno F, Claro RF, Levy RB, Bandoni DH, Ferreira SRG, Monteiro CA. Estimativa de consumo de sódio pela população brasileira, 2002-2003. Rev Saude Publica 2009; 43(2):219-225.

12. Legetic B, Campbell N. Reducing salt intake in the Americas: Pan American Health Organization actions. J Health Commun 2011; 16(Supl. 2):37-48.

13. Wardle J, Steptoe A. The european health and behaviour survey: rationale, methods and initial results from the United Kingdom. Soc Sci Med 1991; 33(8):925-936.

14. Wardle J, Haase AM, Steptoe A, Nillapun M, Jonwutiwes K, Bellisle F. Gender differences in food choice: The contribution of health beliefs and dieting. Ann Behav Med 2004; 27(2):107-116.

15. Tseng M, Devellis RF. Fundamental dietary patterns and their correlates among US whites. $J A m$ Diet Assoc 2001; 101(8):929-932.

16. Neumman AICP, Martins IS, Marcopito LF, Araujo EAC. Padrões alimentares associados a fatores de risco para doenças cardiovasculares entre residentes de um município brasileiro. Rev Panam Salud Publica 2007; 22(5):329-339.

17. Molina MCB, Cunha RS, Herkenhoff LF, Mill JG. Hipertensão arterial e consumo de sal em população urbana. Rev Saude Publica 2003; 37(6):743-750.
18. Brasil. Ministério da Saúde (MS). VIGITEL Brasil 2006: Vigilância de fatores de risco e proteção para doenças crônicas por inquérito telefônico: estimativas sobre frequência e distribuição sociodemográfica de fatores de risco e proteção para doenças crônicas nas capitais dos 26 estados brasileiros e no Distrito Federal em 2006. Brasília: MS; 2007.

19. Moura EC, Neto OLM, Malta DC, Moura L, Silva NN, Bernal R, Claro RM, Monteiro CA. Vigilância de fatores de risco para doenças crônicas por inquérito telefônico nas capitais dos 26 estados brasileiros e no Distrito Federal (2006). Rev Bras Epidemiol 2008; 11(Supl. 1):20-37.

20. Hotchkiss JW, Davies C, Gray L, Bromley C, Capewell S, Leyland AH. Trends in adult cardiovascular disease risk factors and their socio-economic patterning in the Scottish population 19952008: cross-sectional surveys. BMJ Open 2011; 1(1):e000176.

21. Hu J, La Vecchia C, Morrison H, Negri E, Mery L; Canadian Cancer Registries Epidemiology Research Group. Salt, processed meat and the risk of cancer. Eur J Cancer Prev 2011; 20(2):132-139.

22. Grimes CA, Riddell LJ, Nowson CA. The use of table and cooking salt in a sample of Australian adults. Asia Pac J Clin Nutr 2010; 19(2):256-260.

23. Ray S, Kulkarni B, Sreenivas A. Prevalence of prehypertension in young military adults \& its association with overweight \& dyslipidaemia. Indian J Med Res 2011; 134(2):162-167.

24. Sanchez-Castillo CP, Warrender S, Whitehead TP, James WP. An assessment of the sources of dietary salt in a British population. Clin Sci (Lond) 1987; 72(1):95-102.

25. Bleil IS. O padrão alimentar ocidental: considerações sobre a mudança de hábitos no Brasil. Cad Debate 1998; 6:1-25.

26. Instituto Brasileiro de Geografia e Estatística (IBGE). Pesquisa de Orçamentos Familiares, 2008-2009: Avaliação nutricional da disponibilidade domiciliar de alimentos no Brasil. Rio de Janeiro: IBGE; 2010.

27. Melse-Boonstra A, Rexwinkel H, Bulux J, Solomons NW, Weste CE. Comparison of three methods for estimating daily individual discretionary salt intake: 24 hour recall, duplicate-portion method, and urinary lithium-labelled household salt excretion. Eur J Clin Nutr 1999; 53(4):281-287.

28. Reckelhoff JF. Gender differences in the regulation of blood pressure. Hypertension 2001; 37(5):11991208.

29. Anastos K, Charney P, Charon RA, Cohen E, Jones CY, Marte C, Swiderski DM, Wheat ME, Williams S. Hypertension in women: What is really known? Ann Intern Med 1991; 115(4):287-293.

30. Desmond E. Reducing salt: A challenge for the meat industry. Meat Sci 2006; 74(1):188-196.

31. Kiefer I, Rathmanner T, Kunze M. Eating and dieting differences in men and women. J Men's Health Gend 2005; 2(2):194-201. 
32. Iser BPM, Yokota RTC, Bandeira de Sá NN, Moura L, Malta DC. Prevalência de fatores de risco e proteção para doenças crônicas nas capitais do Brasilprincipais resultados do Vigitel 2010. Cien Saude Colet 2012; 17(9):2343-2356.

33. Courtenay WH. Engendering health: a social constructionist examination of men's health beliefs and behaviors. Psychol Men Masculin 2000; 1(1):4-15.

34. Purdy J, Armstrong G, Mcllveen H. The influence of socio-economic status on salt consumption in Northern Ireland. Int J Cons Stud 2002; 26(1):71-80

35. Roos E, Lahelma E, Virtanen M, Prättälä R, Pietinen P. Gender, socioeconomic status and family status as determinants of food behaviour. Soc Sci Med 1998; 46(12):1519-1529.

36. Instituto Brasileiro de Geografia e Estatística (IBGE). Pesquisa de Orçamentos Familiares, 2002-2003: Aquisição alimentar domiciliar per capita. Rio de Janeiro: IBGE; 2004.

37. Barreto SM, Figueiredo RC. Doença crônica, autoavaliação de saúde e comportamento de risco: diferença de gênero. Rev Saude Publica 2009; 43(Supl. 2):3847.

38. Conry MC, Morgan K, Curry P, McGee H, Harrington J, Ward M, Shelley E. The clustering of health behaviours in Ireland and their relationship with mental health, self-rated health and quality of life. BMC Public Health 2011; 11(1):692

39. Tsai J, Ford ES, Li C, Zhao G, Pearson WS, Balluz LS. Multiple healthy behaviors and optimal selfrated health: findings from the 2007 Behavioral Risk Factor Surveillance System Survey. Prev Med 2010; 51(3-4):268-274.

40. Cook NR, Cutler JA, Obarzanek E, Buring JE, Rexrode KM, Kumanyika SK, Appel LJ, Whelton PK. Long term effects of dietary sodium reduction on cardiovascular disease outcomes: observational follow-up of the trials of hypertension prevention (TOHP). BMJ 2007; 334(7599):885-888.

41. Cotta RMM, Batista KCS, Reis RS, Souza GA, Dias G, Castro FAF, Alfenas RCG. Perfil sociossanitário e estilo de vida de hipertensos e/ou diabéticos, usuários do Programa Saúde da Família no município de Teixeiras, MG. Cien Saude Colet 2009; 14(4):12511260.

42. Centers for Disease Control and Prevention (CDC). Sodium intake among adults-United States, 20052006. MMWR Morb Mortal Wkly Rep 2010; 59(24):746-749.

43. Pan American Health Organization (PAHO), World Health Organization (WHO). Prevenção de doenças cardiovasculares pela redução de sal na alimentação: Relatório da Reunião do grupo de especialistas. Washington: PAHO, WHO; 2009.

Artigo apresentado em 01/08/2013

Aprovado em 26/09/2013

Versão final apresentada em 02/10/2013 